\title{
Reactive Power Tracing Index LQP_LT for Voltage Unstable Load Bus Identification in Power Systems
}

\author{
Renuga Verayiah ${ }^{1}$, Azah Mohamed², Syahirah Abd Halim ${ }^{3}$ \\ ${ }^{1}$ Electrical PowerEngineering Department, Universiti Tenaga Nasional, Selangor, Malaysia \\ ${ }^{2,3}$ Centre for Integrated Engineering Systems and Advanced Technologies, Universiti Kebangsaan Malaysia, \\ Selangor, Malaysia
}

\begin{tabular}{l} 
Article Info \\
\hline Article history: \\
Received Apr 21, 2018 \\
Revised May 21, 2018 \\
Accepted Jul 1, 2018 \\
\hline
\end{tabular}

\section{Keywords:}

Undervoltage load shedding Voltage instability

\begin{abstract}
Existing power systems are significantly susceptible to voltage instability problem since such systems are stressed with the huge power transfers across the grids. To guarantee power system stability during stressed conditions, it is important to first identify the voltage unstable load buses to determine appropriate locations for under voltage load shedding. In this study, a new method is proposed for determining weak load bus locations by using reactive power tracing to develop a novel reactive power tracing capable index, named as LQP_LT. The reactive power tracing algorithm is integrated with the LQP_LT index formulation to generate priority ranking list of weak load buses. The LQP_LT index was tested on the 57 bus system and the resulting priority ranking list is found to have successfully determined the weak load buses for load shedding in the test system. Comparison with other stability indices revealed that the LQP_LT has better sensitivity and response towards determining the location of the weakest load bus for under voltage load shedding implementation.
\end{abstract}

Copyright $\odot 2018$ Institute of Advanced Engineering and Science. All rights reserved.

\section{Corresponding Author:}

Azah Mohamed,

Centre for Integrated Engineering Systems and Advanced Technologies,

Universiti Kebangsaan Malaysia,

Selangor, Malaysia.

Email: azah_mohamed@ukm.edu.my

\section{INTRODUCTION}

Voltage instability has been regarded as one of the major cause of power system insecurity. A voltage instability phenomenon takes place when the receiving end voltage decreases well below its normal operating point [1]. The voltage decline in voltage instability framework occurs when the combined generation and transmission system is unable to provide or meet the power demanded by the loads. The imbalance between the generation power and demand power can be due to system outage or limitations in reactive power sources or a combination of both. To prevent power system from wide-spread voltage collapse situation, usually load shedding is applied as the final safety measure in the mitigation plan. Rapid under voltage load shedding at the voltage unstable locations has been found to be economical and effective in preventing the system voltage from declining rapidly. Therefore, identifying the voltage unstable areas in a power system is crucial before implementing under voltage load shedding.

Several methods have been developed to identify the voltage unstable locations in which the recent methods are based on sensitivity analysis and voltage stability index calculation [2]. Sensitivity analysis can be classified into P-V and V-Q analyses. Due to the nonlinear nature of power system operation, the magnitude of the sensitivities for different system conditions does not provide a direct measure of the relative degree of voltage stability. The sensitivity method is considered time-consuming because it requires repetitive power flow solutions and therefore incurs high computational time when solving large networks. 
Another method for identifying voltage unstable locations is by identifying critical lines or critical buses using proximity indicators based on voltage stability index. Many voltage stability indices (VSI) have been developed and can be categorised as bus indices, system indices and line indices [3]. Bus voltage stability indices determine voltage stability of system buses but do not provide any information about the behaviour of the lines, loads or generators which may cause voltage instability in the system. The system VSIs categorized here are not related to the system buses or lines because these indices are not able to determine the weakest bus or line but can only predict the system collapse point [4]. Many line VSIs have been formulated based on the representation of a transmission line in which the discriminant of the voltage quadratic equation for most of the lines is set to be equal or greater than zero, to achieve voltage stability. Some examples of the line VSIs that have been developed are the Line Stability Index (L_mn), fast Volatge Stability Index (FVSI) and the New Voltage Stability Index (NVSI) [5]-[7].

All the VSIs have their advantages and disadvantages and therefore it is not practical to recommend an index as the only one to use. Some indices are suited for on-line studies because of low computational time but these indices tend to be poor predictors of proximity to voltage collapse. On the other hand, some indices give accurate measure of distance to voltage collapse but require high computational time and making them unsuitable for online studies [3]. It is important to note that all the above-mentioned indices have neglected the effect of shunt admittances and reactive power in its computation to predict voltage instability [8]. The application of the indices for determining the voltage unstable locations is also not considered due to lack of information on reactive power tracing. Reactive power flow is required to maintain the voltage to deliver active power through transmission lines. Due to the restructuring of power system operation from regulated to deregulated market, there is a need to provide the service of reactive power support not only for transmission system pricing but also for voltage stability control. In view of more complex and practical power systems, it is important to evaluate the reactive power flow in every transmission line and determine its contribution to voltage stability in a power system. Hence, to develop an accurate voltage collapse indicator for determining the voltage unstable locations, the power flow tracing principle developed by Bialek and improvised power flow tracing method by Niu et al. is applied by considering reactive power flow in this research work [9]-[10].

This paper describes the development of a new stability index that incorporates reactive power tracing computation in the index for determining the weak locations from the voltage instability point of view. The proposed reactive power tracing method does not alter the network size and it can trace the reactive power flow contributed by load buses in a transmission system. The proposed reactive power tracing index LQP_LT is then compared with the other line VSIs such as the Lmn, FVSI and NVSI [5]-[7].

\section{DERIVATION OF REACTIVE POWER TRACKING INDEX LQP_LT}

Load tracing is a task for determining the extraction of power by loads and is the key principle behind the derivation of the reactive power tracing index. Load tracing determines how much line flow and generated power are consumed by a load [11]-[12]. Tracing the power consumption by any load in a system can provide a good indication on the priority of load to be shed first during a contingency. The section describes the development of the proposed reactive power tracing index, LQP_LT for determining the weak load buses for load shedding purpose. The LQP_LT index is derived from the line stability factor, LQP developed by Mohamed et al [13]. Using the concept of reducing a radial network into a single line equivalent circuit, the power flow in a transmission line of a two bus system is considered as shown in Figure 1 [14].

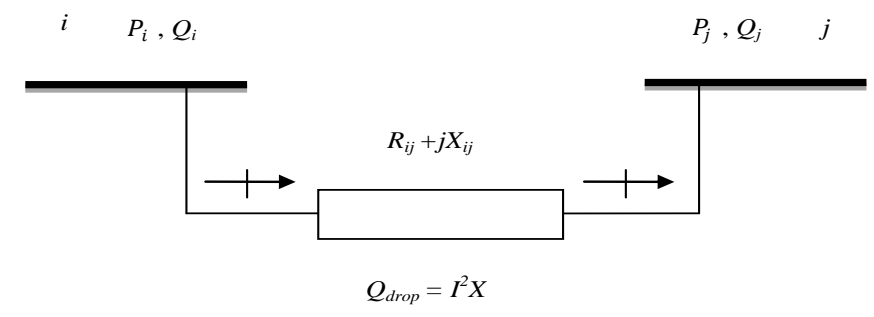

Figure 1. Single line diagram of a two-bus system

By taking node $i$ as the reference node and letting $Q j$ be the the reactive power entering node $j$, the following equation is derived [13]. 


$$
Q_{j}=Q_{i}-Q_{\text {drop }}=Q_{i}-I^{2} X
$$

Thus,

$$
Q_{j}=Q_{i}-\frac{X_{i j}\left(P_{i}^{2}+Q_{i}^{2}\right)}{V_{i}^{2}}
$$

Rearranging (2),

$$
\frac{X_{i j} Q_{i}^{2}}{V_{i}^{2}}-Q_{i}+\left(Q_{j}+\frac{X_{i j} P_{i}^{2}}{V_{i}^{2}}\right)=0
$$

Expressing (3) into the quadratic form and solving for $Q_{i}$,

$$
Q_{i}=\frac{1 \pm \sqrt{1^{2}-4\left(\frac{X_{i j}}{V_{i}^{2}}\right)\left(\frac{Q_{j}+P_{i}^{2} X_{i j}}{V_{i}^{2}}\right)}}{2\left(\frac{X_{i j}}{V_{i}^{2}}\right)}
$$

Thus, for real roots of $Q_{i}$, the following equation must be satisfied.

$$
1-4\left(\frac{X_{i j}}{V_{i}^{2}}\right)\left(Q_{j}+\frac{X_{i j} P_{i}^{2}}{V_{i}^{2}}\right)=0
$$

The condition in (5) will lead to imaginary roots of $Q_{i}$ if it is not satisfied. From (5), it can also be observed that the sending end voltage, $V_{i}$ has to be zero in order for the reactive power, $Q_{i}$ to become a real quantity. Therefore, at this point when the sending end voltage, $V_{i}$ become zero and the condition in (5) is not satisfied, it implies that voltage collapse scenario have already occured. From (5), the LQP index is derived and expressed as [13],

$$
L Q P=4\left(\frac{X_{i j}}{V_{i}^{2}}\right)\left(Q_{j}+\frac{X_{i j} P_{i}^{2}}{V_{i}^{2}}\right)
$$

The LQP index has a value between 0 to 1.0 and that if the value of LQP index is close to 1.0, it indicates proximity to voltage collapse.

Since reactive power flow plays a crucial role in maintaining a voltage within a stable region, it is important to trace the reactive power flow in a line that will be consumed by a load. The significance of tracing reactive power flow in every line due to load will in fact indicate which load buses in the system that causes highest congestion level to a transmission line. With this concept, tracing the LQP index contributed by a certain load in a system can be derived. Equation (4) has shown that reactive power can be traced in a line as a summation of individual load components by identifying the power fraction contribution. Applying (4) and (5) for reactive power tracing, into (6) for line $l-m$ as summation of individual load components, the Reactive Power Tracing Index, LQP_LT is derived. The following sections show the detailed steps to derive LQP_LT index.

To successfully obtain the traced LQP_LT index, the receiving end reactive power fraction, $x_{l m}^{i}$ extracted by $i$-th load in line $l-m$ need to be quantified. To perform this, the downstream looking algorithm which considers the nodal balance outflows is implemented. Applying this concept by Bialek, the total nodal reactive power, $Q_{i}$ is expressed as [9],

$$
\begin{aligned}
& Q_{i}=\sum_{j \in \alpha_{i}^{(d)}}\left|Q_{l-m}\right|+Q_{L i}=\sum_{j \in \alpha_{i}^{(d)}} x_{l m}^{i} Q_{j, l-m}+Q_{L i} \\
& \text { for } i=1,2,3 \ldots, \mathrm{n}
\end{aligned}
$$

where $\alpha_{i}^{(d)}$ is the set of nodes supplied directly from node $i$.

Thus, the reactive power flowing out from node $i$ in the relevant lines $l-m$, is noted as $Q_{l-m}$. On the other hand, $Q_{L i}$ is the reactive load at node $i, Q_{j, l-m}$ is the receiving end reactive power extracted by reactive power faction $x_{l m}^{i}=\left|Q_{j, l-m}\right| / Q_{i}$. Rearranging (7), it can be re-written as, 


$$
Q_{i}-\sum_{j \in \alpha_{i}^{(d)}} x_{l m}^{i} Q_{j, l-m}=Q_{L i}
$$

or as multiplication of downstream distribution admittance matrix [9],

$$
A_{d} Q=Q_{L}
$$

where $A_{d}$ is the ( $\left.n \times n\right)$ downstream distribution matrix, $Q$ is the vector of nodal through reactive power flows and $Q_{L}$ is the vector of nodal reactive demands. The $(l-m)$ element of downstream distribution matrix, $A_{d}$ is equal to,

$$
\begin{aligned}
& {\left[A_{d}\right]_{l m}=\left\{\begin{array}{c}
1 \\
-x_{l m}^{i} \\
0
\end{array}=-\left|Q_{j, l-m}\right| / Q_{i}\right.} \\
& \text { for } l-\mathrm{m} \in \alpha_{\mathrm{i}}^{(\mathrm{d})} \\
& \text { for } l-m=i
\end{aligned}
$$

From (10), if $A_{d}$ exists, then $Q=A_{d}^{-1} Q_{L}$ and its $n$-th element can be written as:

$$
\begin{aligned}
& Q_{\mathrm{i}}=\sum_{\mathrm{k}=1}^{\mathrm{n}}\left[\mathrm{A}_{\mathrm{d}}^{-1}\right]_{\mathrm{ik}} Q_{\mathrm{Lk}} \\
& \text { for } i=1,2, \ldots, \mathrm{n}
\end{aligned}
$$

Equation (11) shows how the nodal reactive power, $Q_{\mathrm{i}}$ is distributed between all the loads in the system. Bialek has shown that the same nodal reactive power, $Q_{\mathrm{i}}$ is equal to the sum of the load demand at node $i$ and all the outflows in lines leaving the node [9]. Hence, the reactive power outflow in line $l-m$ from node $i$ can be calculated using the proportional sharing principle shown as,

$$
\left|Q_{l-m}\right|=\left(\frac{\left|Q_{j, l-m}\right|}{Q_{i}}\right) \sum_{k=1}^{n}\left[A_{d}^{-1}\right]_{i k} Q_{L k}
$$

From here, the traced reactive power allocation to each line due a particular load component is implemented into the $L Q P$ index and named as $L Q P_{-} L T_{l m} . L Q P_{-} L T_{l m}$ can now be written as:

$$
L Q P_{-} L T_{l m}=L Q P_{l m}^{L 1}+L Q P_{l m}^{L 2}+L Q P_{l m}^{L 3}+\cdots+L Q P_{l m}^{L n}
$$

Expanding (13) to incorporate the traced reactive power tracing contributed by load components in individual line $l-m$, the following is derived.

$$
L Q P_{-} L T_{l m}=4\left(\frac{X_{i j}}{V_{i}^{2}}\right)\left(Q_{l m}^{L 1}+\frac{X P_{i}^{2}}{V_{i}^{2}}\right)+4\left(\frac{X_{i j}}{V_{i}^{2}}\right)\left(Q_{l m}^{L 2}+\frac{X P_{i}^{2}}{V_{i}^{2}}\right)+\cdots+4\left(\frac{X_{i j}}{V_{i}^{2}}\right)\left(Q_{l m}^{L n}+\frac{X P_{i}^{2}}{V_{i}^{2}}\right)
$$

Finally, the total traced LQP_LT of line $l-m$ due to the $i$-th load of reactive power, $Q_{L i}$ is generalised and written as follows,

$$
L Q P_{-} L T_{l m}=4\left(\frac{X_{i j}}{V_{i}^{2}}\right)\left(\left[\sum_{i=1}^{L n} Q_{l m}^{L i}\right]+\frac{X_{i j} P_{i}^{2}}{V_{i}^{2}}\right)
$$

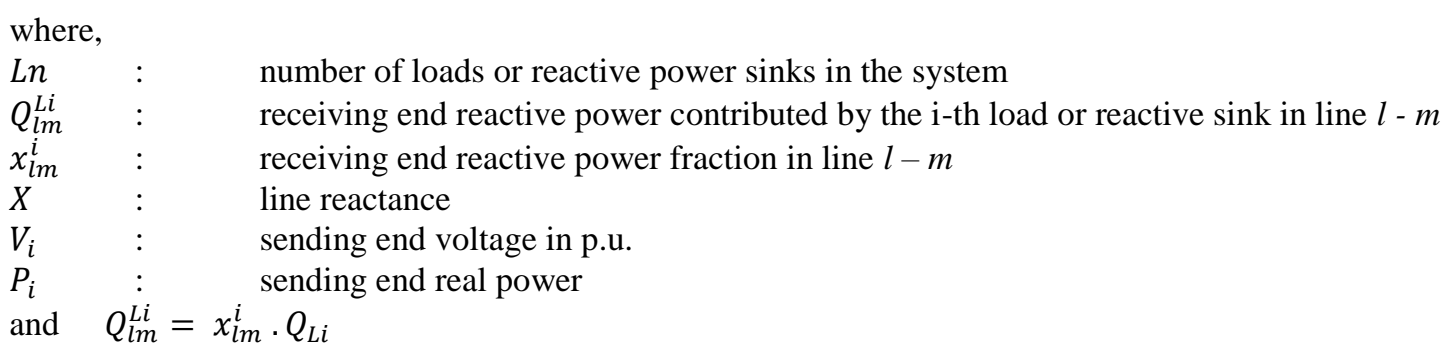

\section{REACTIVE POWER TRACING ALGORITHM}

A reactive power tracing algorithm is developed in MATLAB to calculate the proposed reactive power tracing index, LQP_LT and ranking the index for identifying the weak lines in a power system. The overall implementation steps of the reactive power tracing algorithm are described as follows: 
Step 1: Input data of the test power system.

Step 2: Obtain the base case power flow solution for the power system using the MATLAB based MATPOWER load flow program.

Step 3: Apply a disturbance event, such as single line outage, double line outage with and without load increase in the system.

Step 4: Calculate new reactive power in each line at both sending and receiving end buses due to reactive power generated by shunt admittance, $Q_{\text {shunt }}$ connected to each bus. Equations (16) and (17) are used to obtain the new reactive power injections considering the shunt admittances.

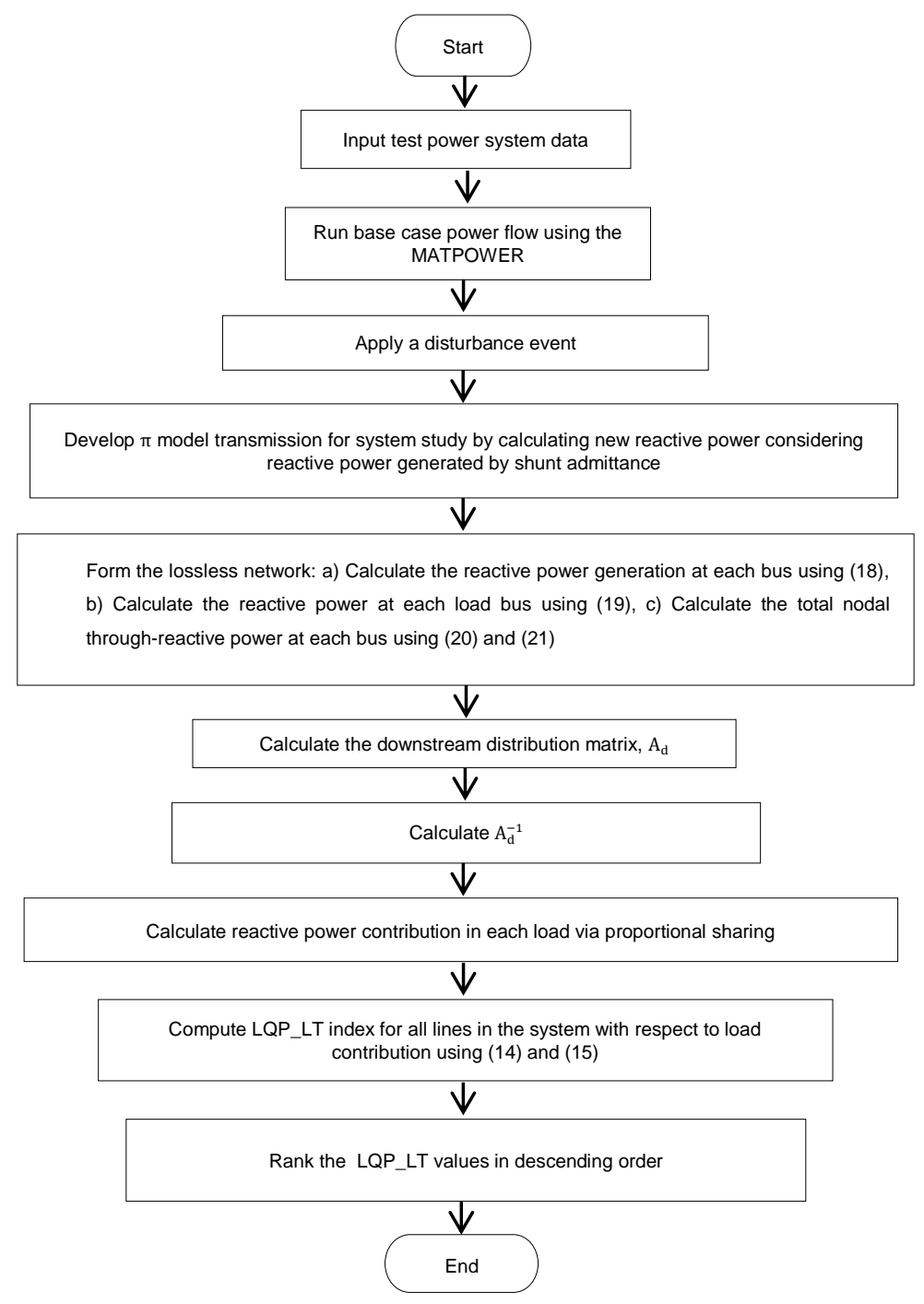

Figure 2. Implementation steps of LQP_LT index computation

$$
\begin{aligned}
& Q_{\text {sending,new }}=Q_{\text {sending }}+G_{Q i} \\
& Q_{\text {receiving,new }}=Q_{\text {receiving }}-G_{Q j}
\end{aligned}
$$

Step 5: Complete the $\pi$ model network by considering the following calculations. The network is now considered as lossless since the shunt admittances has been attributed to sending end buses as shown in Step 4 .

a) Calculate the reactive power generation at each bus that is equal to total generated power and attributed shunt admittances. 


$$
Q_{\text {gen,new }}=Q_{\text {gen }}+G_{Q i}
$$

b) Calculate the reactive power at each load bus that is equal to total reactive load and attributed shunt admittances.

$$
Q_{\text {load,new }}=Q_{\text {load }}-G_{Q j}
$$

c) Calculate the total nodal through-reactive power at each bus, $i$.

$$
\begin{aligned}
& Q_{i}=Q_{\text {gen,new }}+Q_{\text {receiving,new }} \\
& Q_{i}=Q_{\text {load,new }}+Q_{\text {sending,new }}
\end{aligned}
$$

Step 6: Form the downstream distribution matrix, $A_{d}$ by using the downstream looking algorithm as described by (9) to (12).

Step 7: Calculate the inverse of downstream distribution matrix, $A_{d}^{-1}$.

Step 8: Calculate the reactive power contribution in each line as given in (12).

Step 9: Calculate the LQP_LT index for all lines in the system using (14) and (15).

Step 10: Rank the LQP_LT index values in descending order.

The implementation steps of the reactive power tracing algorithm and the LQP_LT index calculation for every transmission line in a power system is also described in terms of a flow chart as shown in Figure 2.

\section{TEST RESULTS}

To evaluate the effectiveness and the accuracy of the proposed reactive power tracing index, LQP_LT in determining the weak buses in a power system, it is compared with other other voltage stability indices, namely, FVSI, Lmn and NVSI [5]-[7]. Similar contingency scenarios for the single line and double contingency are simulated on the IEEE 57 bus test system using the MATPOWER power flow analysis program. From the MATPOWER power flow results, the LQP_LT index values are computed for all the buses in the selected contingency scenario and contingency ranking is performed. The voltage stability indices, FVSI, Lmn and NVSI are also computed and ranking results from these indices are obtained. The ranking results obtained from the LQP_LT index and the other voltage stability indices are then analyzed and compared. The following sections describe the formulation of voltage stability indices, FVSI, Lmn and NVSI. The line stability index, known as Lmn is expressed as [5],

$$
L m n=\frac{4 Q_{r} X}{\left[\left|V_{S}\right| \sin (\theta-\delta)\right]^{2}}
$$

where $X$ is the line reactance, $Q_{r}$ is the reactive power at the receiving end, $V_{s}$ is the sending end voltage, $\theta$ is line impedance angle and $\delta$ is the angle difference between the supply voltage and the receiving voltage. $\mathrm{Lmn}$ is used for predicting voltage instability such that if Lmn is less than 1.00, the power system is considered stable, otherwise the system is unstable. The FVSI is expressed as [6],

$$
F V S I=\frac{4 Z^{2} Q_{r}}{V_{S}^{2} X}
$$

where $Z$ is the line impedance, $X$ is the line reactance, $Q_{r}$ is the reactive power flow at the receiving end and $V_{s}$ is the sending end voltage. The line that gives index value close to 1.00 will be the most critical line and may lead to system wide voltage instability. The NVSI [7] is expressed as,

$$
N V S I=\frac{2 X \sqrt{\left(P_{j}^{2}+Q_{j}^{2}\right)}}{2 Q_{r} X-V_{i}^{2}}
$$

where $X$ is the line reactance, $Q_{r}$ is the reactive power flow at the receiving end and $V_{i}$ is the sending end voltage. For NVSI value less than 1.00 , the system is considered to be in voltage stable condition. To test the effectiveness and performance of the LQP_LT index on the test systems, two load conditions have been considered; base case and heavily loaded conditions. The results of the LQP_LT index for identifying the weak load buses and weak lines are presented in the following sections. 


\subsection{LQP_LT Index Computation for Base Case Condition}

Table 1 shows the first five highest $\sum_{L o a d} L Q P_{-} L T$ index values obtained for the IEEE 57 bus system. The five buses with high $\sum_{L o a d} L Q P_{-} L T$ index values for the 57 bus system are bus 31 followed by bus 18 , bus 42 , bus 25 and bus 53 . Close monitoring would be needed at these weak or voltage unstable buses as further increase in load demand may cause insufficient reactive power to maintain the bus voltage close to its nominal value.

Table 1. Five Highest LQP_LT Index Values at the Load Buses in the IEEE 57 bus System for

\begin{tabular}{cccccc}
\multicolumn{6}{c}{ Base Case Condition } \\
\hline Load bus & 31 & 18 & 42 & 25 & 53 \\
\hline$\sum_{\text {Load }}$ LQP_LT & 0.8264 & 0.7920 & 0.7853 & 0.7689 & 0.7365 \\
\hline
\end{tabular}

The capability of the LQP_LT index is not restricted in tracing weak load buses only, but it can also trace and rank the weak lines affected by those load buses. Table 2 shows the five highest LQP_LT index, $\sum_{\text {Lines }} L Q P_{-} L T$, traced for the lines in the IEEE 57 bus system. It is important to note that the summation of the LQP_LT values computed for each line, $\sum_{L i n e s} L Q P \_L T$, in the system consist of individually traced, $L Q P_{-} L T_{l-m \text { (load) }}$ contributed by the load buses connected to the line. Thus, the $\sum_{\text {Lines }} L Q P \_L T$ index value computed for each line is possible to exceed 1.0. From Table 2, it was found that the results of the $\sum_{\text {Lines }} L Q P_{-} L T$ for the base case in the IEEE 57 bus system indicate that line connecting from bus 24 to bus 25 has the highest congestion level and need close monitoring for any increase in the load demand. Evaluation from the base case analysis have demonstrated that tracing the reactive power fraction contribution by the load buses to the transmission lines using $\sum_{\text {Lines }} L Q P_{-} L T$ index formulation and ranking system, would enable the system operator to identify the exact location of weak lines that exert high reactive power flow in the lines. Thus, any corrective or preventive action to maintain system stability can be executed effectively and accurately.

Table 2. Five highest $\sum_{\text {Lines }} L Q P \_L T$ values for the base case of IEEE 57 bus system

\begin{tabular}{ccc}
\hline From bus & To bus & $\sum_{\text {Lines }}$ LQP_LT \\
\hline 24 & 25 & 1.6371 \\
8 & 9 & 1.6218 \\
4 & 18 & 1.4457 \\
11 & 41 & 1.3230 \\
4 & 18 & 1.2727 \\
\hline
\end{tabular}

\subsection{LQP_LT Index Computation for Heavily Loaded System}

To test the effectiveness of LQP_LT index in identifying the weak load buses and weak lines in the IEEE 57 bus system, the loads are increased in stages until the power flow diverges. For the IEEE 57 bus system, the power flow diverges when the loading at all the load buses are increased by $57.9 \%$ or by a loading factor of 1.58 from the base case load. Figure 2 shows the $\sum_{\text {Lines }} L Q P \_L T$, recorded at the 5 most critical lines for the IEEE 57 bus system. From the figure, it was found that LQP_LT summation on the lines, $\sum_{\text {Lines }} L Q P \_L T$, for the IEEE 57 bus system increases as the load demand in all load buses are increased to a maximum of $57.9 \%$ from the base values. It is obvious that line connecting bus 1 to bus 16 has the highest $\sum_{\text {Lines }} L Q P_{-} L T$, that is, 10.2585 . This indicates that the line connecting bus 1 to bus 16 has the highest congestion level, or shortage of transmission capacity to cater for the increased load demand.

On the other hand, the ranking results of $\sum_{L o a d} L Q P_{L T}$ for the IEEE 57 bus system is shown in Figure 3. The highest $\sum_{L o a d} L Q P_{L T}$ index is obtained at bus 31 followed by bus 18, 25, 42 and bus 53 . This indicates that these buses are the weak load buses in the system, and have contributed to high congestion level in the critical lines shown in Figure 2. It can be concluded that bus 31, followed by bus 18, bus 25, bus 42 and bus 53 are the suitable locations for load shedding execution. 


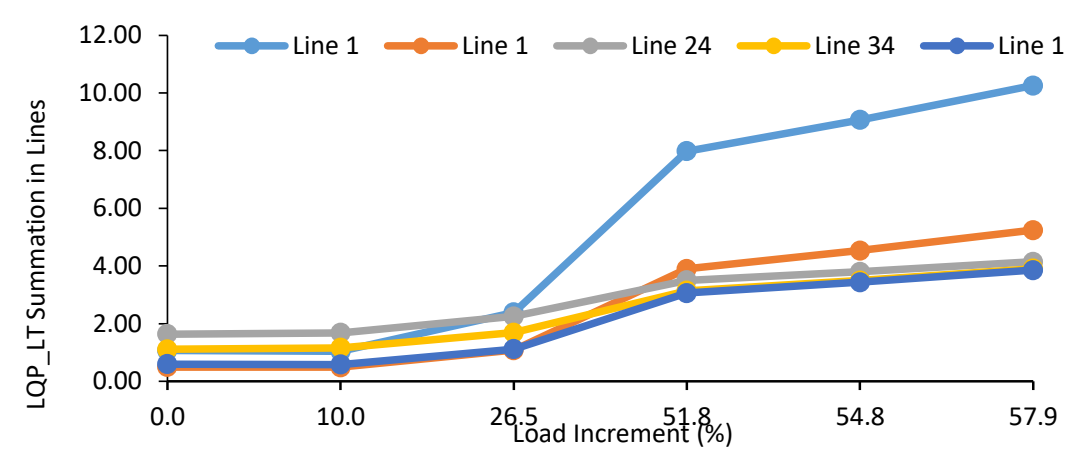

Figure 2. Performance of $\sum_{\text {Lines }} L Q P_{-} L T$ for 5 critical lines in the IEEE 57 bus system for increased load condition

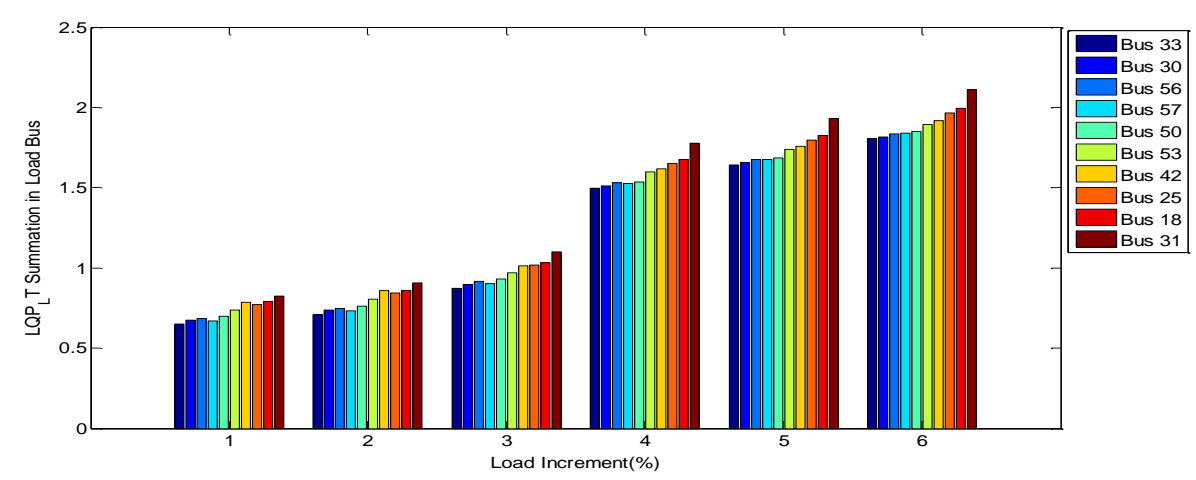

Figure 3. LQP_LT index, $\sum_{\text {Load }} L Q P_{-} L T$, computed for load buses with loading increment

\subsection{Comparison of LQP_LT Index with NVSI, Lmn and FVSI}

To evaluate and validate the weak load bus identification using the proposed $\sum_{L o a d} L Q P \_L T$, a comparison was made for the heavily loaded IEEE 57 bus system using NVSI, FVSI and Lmn indices. To maintain consistency in this comparison study, the maximum loading factor for the test system is considered to evaluate the performance of the NVSI, FVSI and Lmn indices in determining the weak lines and weak load buses. Table 3 shows the ranking results obtained by using the NVSI, FVSI, Lmn and LQP_LT indices at the maximum loading of $57.9 \%$ for the IEEE 57 bus system. The results are ranked from highest index to the lowest index to indicate the critical lines and the weak load buses in the system.

For the FVSI, NVSI and Lmn indices, the receiving end bus for each critical line identified is taken as the weak load bus. The 10 critical lines for the test system are shown in Table 3. Evaluation on the index performance for the system shows that Lmn and NVSI index indicated bus 16 as the weakest bus, while LQP_LT index traced bus 31 as the weakest bus and the FVSI index indicated bus 49 as the weakest bus. Subsequent ranking order for the weak load buses varies for all the indices. The FVSI index indicated line connecting from bus 13 to bus 49 as the most critical line, where else LQP_LT, LMN and NVSI indices have indicated line connecting from bus 1 to bus 16 as the most critical line. From the results shown in Table 3, the NVSI, FVSI, LMN and LQP_LT indices give different ranking order of the critical lines but there are similarities in the critical lines detected. The difference in results is due to the variation in the index formulation. For example, NVSI and LQP_LT indices considers the active and reactive powers in its formulation, where else the LMN and FVSI indices consider only the receiving end reactive power.

The results also show that the common critical line identified by $\sum_{L i n e s} L Q P \_L T$ index and other indices namely, NVSI, FVSI and LMN is the line connecting bus 13 to bus 49 as shown in Table 3. The $\sum_{\text {Lines }} L Q P_{-} L T$ for this line, which is $\sum_{13-49} L Q P_{-} L T$ has been derived from the reactive power fraction contribution of all the load buses in the system as shown in Figure 4. Load bus 23 has the most contribution in this line with the highest index value of 0.0549 , and then followed by load bus 31 with a value of 0.0464 and bus 25 with a value of 0.0431 . The other indices such as FVSI, NVSI and LMN are not able to reveal much information on the participation of the load buses in the system as compared to the tracing method. 
Table 3. Critical lines and weak load buses ranking at maximum loading

\begin{tabular}{|c|c|c|c|c|c|c|c|c|c|c|c|c|c|}
\hline \multirow[b]{2}{*}{$\begin{array}{c}\text { Ranking } \\
\text { No. }\end{array}$} & \multicolumn{3}{|c|}{ FVSI } & \multicolumn{3}{|c|}{ NVSI } & \multicolumn{3}{|c|}{ LMN } & \multicolumn{4}{|c|}{ LQP_LT } \\
\hline & $\begin{array}{l}\text { From } \\
\text { Bus }\end{array}$ & $\begin{array}{c}\text { To } \\
\mathrm{Bu} \\
\mathrm{s}\end{array}$ & FVSI & $\begin{array}{c}\text { From } \\
\text { Bus }\end{array}$ & $\begin{array}{c}\text { To } \\
\mathrm{Bu} \\
\mathrm{s}\end{array}$ & NVSI & $\begin{array}{c}\text { From } \\
\text { Bus }\end{array}$ & $\begin{array}{c}\text { To } \\
\mathrm{Bu} \\
\mathrm{s}\end{array}$ & LMN & $\begin{array}{c}\text { From } \\
\text { Bus }\end{array}$ & $\begin{array}{c}\text { To } \\
\text { Bus }\end{array}$ & $\sum_{\text {Lines }} L Q H$ & $\sum_{\text {Load }} L Q 1$ \\
\hline 1 & 13 & 49 & 0.341 & 1 & 16 & 0.486 & 1 & 16 & 0.694 & 1 & 16 & 10.259 & 31 \\
\hline 2 & 34 & 32 & 0.266 & 34 & 32 & 0.373 & 23 & 24 & 0.667 & 1 & 15 & 5.240 & 18 \\
\hline 3 & 24 & 25 & 0.217 & 24 & 25 & 0.360 & 12 & 10 & 0.579 & 24 & 25 & 4.145 & 25 \\
\hline 6 & 39 & 57 & 0.198 & 13 & 49 & 0.314 & 4 & 5 & 0.513 & 12 & 17 & 3.786 & 50 \\
\hline 7 & 40 & 56 & 0.191 & 1 & 17 & 0.295 & 11 & 43 & 0.511 & 4 & 18 & 2.834 & 57 \\
\hline 8 & 12 & 13 & 0.177 & 11 & 41 & 0.293 & 12 & 13 & 0.492 & 11 & 41 & 2.650 & 56 \\
\hline 9 & 50 & 51 & 0.175 & 4 & 18 & 0.269 & 48 & 48 & 0.456 & 3 & 2 & 2.103 & 30 \\
\hline 10 & 43 & 41 & 0.170 & 39 & 57 & 0.235 & 13 & 49 & 0.332 & 13 & 49 & 1.947 & 33 \\
\hline
\end{tabular}

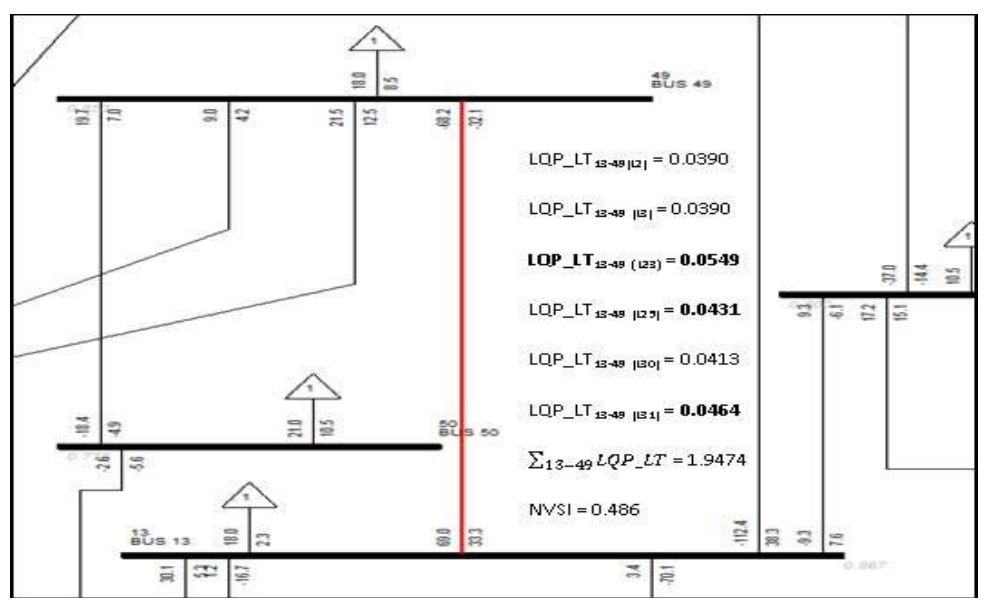

Figure 4. Traced LQP_LT, NVSI, FVSI and LMN index on common critical line (line connecting from bus 13 to bus 49)

\section{CONCLUSION}

The performance of the proposed reactive power tracing index, LQP_LT for determining the voltage unstable areas in a power system has been validated by comparing it with the existing indices such as FVSI, NVSI and Lmn and the IEEE 57 bus test system is used for the comparative analysis. The reactive power tracing applied via LQP_LT index have created a useful ranking list of weak load buses. Comparison of results obtained by LQP_LT index with stability indices NVSI, FVSI and Lmn showed that there is similarity in the determined critical lines and critical load buses. However, the ranking order varies for all the other indices. The drawback of the existing indices as compared to LQP_LT index is that these indices could not exactly indicate the weak load buses for any corrective actions to be taken by system operators. In the literature, choosing the receiving end buses of a critical line located by NVSI, FVSI and Lmn index is a common practice but less accurate. This is proven in this study, since the results obtained have strongly showed that the receiving end buses of any critical line may not be the actual weak bus. Thus, the LQP_LT index which incorporates the power tracing capability is a new index for accurate weak load bus detection in a critical power system. The reactive power tracing method and the proposed LQP_LT index developed in MATLAB can be easily used for online applications due to its simple calculation and robustness.

\section{REFERENCES}

[1] Zamani MK, Musirin I, Omar MS, Suliman SI, Ghani NA, Kamari NA. Gravitational Search Algorithm Based Technique for Voltage Stability Improvement. Indonesian Journal of Electrical Engineering and Computer Science. 2018; 9(1): 123-130.

[2] Nor AF, Sulaiman M, Kadir AF, Omar R. Voltage Instability Analysis for Electrical Power System Using Voltage Stabilty Margin and Modal Analysis. Indonesian Journal of Electrical Engineering and Computer Science. 2016; 3(3): 655-622. 
[3] Modarresi J, Gholipour E. A Comprehensive Review of the Voltage Stability Indices. Renewable and Sustainable Energy Reviews. 2016; 63: 1- 12.

[4] Berizzi A. The Italian 2003 Blackout. IEEE Power Engineering Society General Meeting. Denver. 2004; 2 : 1673-1679.

[5] Moghavvemi M, Omar FM. 1997. New Method for Indicating Voltage Stability Condition in Power System. Proceedings of IEEE International Conference on Power Engineering. Singapore. 1997; 223-221.

[6] Musirin I, Abdul Rahman TK. Estimating Maximum Loadability for Weak Bus Identification Using FVSI. IEEE Power Engineering Review. 2002; 22(11): 50-52.

[7] Kanimozhi R, Selvi K. A Novel Line Stability Index for Voltage Stability Analysis and Contingency Ranking in Power Systems using Fuzzy Based Load Flow. Journal of Electrical Engineering Technology. 2013. 8(4): 694-703.

[8] Li S. Sensitivity Model of L Index for Steady-State Voltage Stability of Wind Power Systems with Doubly Fed Induction Generators. Electrical Power Components and Systems. 2016; 44(18): 2017-2024.

[9] Bialek J. Tracing the flow of Electricity. IEEE Proceedings - Generation, Transmission and Distribution. 1996; 143(4): 313-320.

[10] Niu R, Zeng Y, Cheng M, Wang X. Study on Load Shedding Model based on Improved Power Flow Tracing Method in Power System Risk Assessment. 4th International Conference on Electric Utility Deregulation, Restructuring and Power Technologies (DRPT). Shandong. 2011; 45-50.

[11] Zulkiffli AH, Musirin I. Optimal Fuzzy Inference System Incorporated with Stability Index Tracing: An Application for Effective Load Shedding. Expert Systems with Applications. 2014; 41(4): 1095-1103.

[12] Zamani MK, Musirin I, Hassan H, Shaaya SA, Sulaiman SI, Ghani NA, Suliman SI. Active and Reactive Power Scheduling Optimization using Firefly Algorithm to Improve Voltage Stability Under Load Demand Variation. Indonesian Journal of Electrical Engineering and Computer Science. 2018; 9(2): 365-372.

[13] Mohamed A, Jasmon GB, Yusoff S. A Static Voltage Collapse Indicator using Line Stability Indicators. Journal of Industrial Technology. 1989; 7(1): 73- 85.

[14] Jasmon GB, Lee LHCC. Distribution Network Reduction for Voltage Stability Analysis and Loadflow Calculations. International Journal of Electrical Power and Energy Systems. 1991; 13 (1): 9-13. 